\title{
Full Width-Half Maximum Characteristics of FBG for Petroleum Sensor Applications
}

\author{
Hisham K. Hisham \\ Electrical Engineering Department, University of Basrah, Basra, Iraq
}

Correspondence

*Hisham K. Hisham

Electrical Engineering Department,

University of Basrah, Basra, Iraq

Email: Husham_kadhum@yahoo.com

\begin{abstract}
In this paper, we have analyzed the full width-half maximum (FWHM) response of a uniform fiber Bragg grating (FBG) for petroleum sensor applications numerically. The analysis; which is depending on parameters such as the gratings length $(\mathrm{Lg})$ and the amplitude of an induced index profile $(\Delta \mathrm{n})$ change have successfully investigated using MATHCAD software. From the numerical simulation, there is an effect of the $\mathrm{Lg}$ and the $\Delta \mathrm{n}$ on the FWHM response. Results show the Lg value that effect the FWHM response is $\leq 7 \mathrm{~mm}$. In contrast, results show that there is no effect of temperature on the FWHM response. In light of the increasing demand for a wide FWHM band within the applications of optical sensor technology, the results obtained are very important, especially in the field of petroleum industry.
\end{abstract}

Keywords: Fiber Bragg grating (FBG), full width-half Maximum (FWHM), optical sensor technology, petroleum applications.

\section{INTRODUCTION}

Recent years of the last century has seen an important discovery has contributed significantly to the evolution of optical sensor technology; the photosensitivity in optical fibers; resulted in a huge fiber-optic revolution led to the emergence of a new type of fiber components, known as a fiber Bragg grating (FBG) [1, 2]. Where, they can do a lot of the basic operations, such as transmission, reflection, amplification and filtering in communications, in addition to the effective impact in the field of fiber optic sensor (FOS) technology [3].

For years, the petroleum industry has been adopted heavily on conventional electronic sensors (CESs) that are used for distances up to several kilometers inside the Earth's surface to observe a set of an important parameters along the oil well such as pressure and temperature $[4,5]$. However, due to harsh and extreme used environmental conditions such as high temperature and geological factors as a result of the lower layer structure of the Earth, the mechanical pressure on the structural components of these devices makes them suffer from several restrictions such as the effect of the electrical wear, increasing the error rate, decreased the reliability level and the electromagnetic interference (EMI) due to the influence of seismic activities [1]. Moreover, increasing the complexity of monitoring and sensing process due to the large number of devices used in the well bottom which making its reliable is very difficult [1]

In contrast, the accelerated development in the field of FOS technology has contributed to the possibility of expanding the limits of its application to include the FBG due to their unique advantages unparalleled compared to traditional sensor technologies $[1,2]$. In addition to its high ability for measuring the varying in the temperature and the pressure on thousands of points using a single FBG; what is more, it is able to automatically track all system details such as pipelines, flow lines, oil wells with high efficiency $[1,2]$.

Physically, the FBG acts as a wavelength-dependent stop band optical filter as a result of a permanent change in the refractive index of the fiber core [6, 7]. When the travelling mode conditions have satisfied (i.e. Bragg condition), a new rejection window has obtained as results of the constructive interference of the reflected wavelengths. This window is known as the Bragg wavelength, $\lambda_{B}$, which is dependent on the fiber's grating period, $\Lambda$ and its refractive index, $n_{\text {eff }}[1,2]$. Thereby, any changes on the $\Lambda, n_{\text {eff }}$ or in the physical model will cause a shift in the Bragg wavelength [8]. These behaviors of the FBGs parameters will make it imperative, very useful and indispensable sensed tools for many important applications [1, 2].

Basically, the important two parameters that characterize the FBG behavior, namely, are the $n_{\text {eff }}$ and the $\Lambda$. Where, any change in their values lead to a shift

This is an open access article under the terms of the Creative Commons Attribution License, which permits use, distribution and reproduction in any medium, provided the original work is properly cited.

(C) 2020 The Authors. Published by Iraqi Journal for Electrical and Electronic Engineering by College of Engineering, University of Basrah. 
in the $\lambda_{B}[1,2]$. For a uniform FBG as in Fig. 1 , the $\Lambda$ value stay constant throughout the total gratings length $L_{g}$ and the reflected light will be at its maximum at the $\lambda_{B}$ point $[1,2]$.

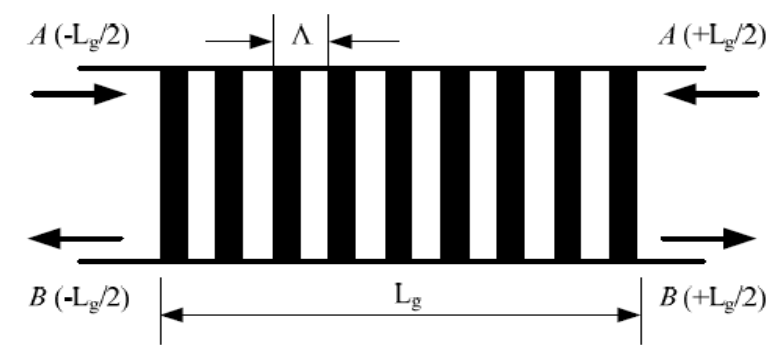

Fig. 1: Uniform fiber Bragg gratings $[1,2]$

Due to their advantages, there is an increasing demand for using a FBG in FOS technology in petroleum applications [9-12]. FBG is intrinsically passive and safe, highly sensitivity, EMI-immune, makes them an indispensable for many applications of sensor fields [1, 2].

Due to the highly wavelength-dependence of the FBG structure, therefore their characteristics_can be easily adjusted by controlling the FBG parameters [1, 2]. Among the most important characteristics of the FBG is the narrow bandwidth $[1,2]$. However, some applications in the petroleum industry such as the seismic and the volcanic vibration require a wide bandwidth range. [13-19]. Therefore, the study of the effect of the FBG parameters on the gratings bandwidth is very important especially for the downhole sensor applications, where temperature and pressure is very high which requires a high-precision monitoring [20, 21].

There are several definitions of the FBG bandwidth [1, 2]. However, the most reliable one is that the criterion which is obtained by calculating the width between the first zeros on both sides of the spectral FBG reflectivity and is defined by the Full Width Half Maximum (FWHM) spectra. The FWHM indicates the bandwidth with a $50 \%$ from the maximum reflectivity $[1,2]$. In this paper, a method based on investigated the effects of FBG parameters on the spectral reflectivity are used for controlling the FWHM response as a sensor tool for petroleum applications. The control parameters selected is the grating length $\left(L_{g}\right)$, the amplitude of the refractive index change $(\Delta n)$ and the temperature variations.

This paper is organized as follows. The basic equations that explain the behavior of light propagation through a FBG is presented in Section II, where the aim is to drive the reflectivity of a uniform FBG model. In Section III, the main mechanisms that used in FBG as a sensor tool are explained. The results and discussions are presented in Section V. Finally, the conclusions are given in Section VI.

\section{THEORY}

One of the best ways that have been used to explain the behavior of light propagation through a waveguide is the coupled mode theory [1, 2, 19, 22]. By assuming a linear combination for the propagate modes, the electric field is given by $[1,19,22]$

$$
E(z)=\sum_{k}\left[A_{k}^{+} \exp \left(-j \beta_{k} z\right)+A_{k}^{-} \exp \left(j \beta_{k} z\right)\right\rfloor \mathrm{E}_{k}
$$

In Eq. $1, A_{k}^{+}, A_{k}^{-}, \beta_{k}$ and $\mathrm{E}_{k}$ are represents the amplitudes of kth mode in the $+\mathrm{z}$ and $-\mathrm{z}$ directions, the propagation constant and the modal field, respectively. By using the coupled mode theory, the electric field equations can be derived as $[1,2,19,22]$

$$
\begin{aligned}
& \frac{d A_{k}^{+}}{d z}=-j \sum_{m}\left\{A_{m}^{+} C_{m k} \exp \left[-j\left(\beta_{m}-\beta_{k}\right) z\right]+\right. \\
& \left.A_{m}^{-} k_{m k} \exp \left[j\left(\beta_{m}+\beta_{k}\right) z\right]\right\} \\
& \frac{d A_{k}^{-}}{d z}=j \sum_{m}\left\{A_{m}^{+} C_{m k} \exp \left[-j\left(\beta_{m}+\beta_{k}\right) z\right]+\right. \\
& A_{m}^{-} k_{m k} \exp \left[j\left(\beta_{m}-\beta_{k}\right) z\right]
\end{aligned}
$$

Under the condition of a uniform single-mode fiber Bragg gratings (FBGs), Equations (2) and (3) are simplified to $[1,2,19,22]$

$$
\begin{gathered}
\frac{d A_{k}^{+}}{d z}=-j \hat{\sigma} A_{k}^{+}(z)-j \kappa^{*} A_{k}^{-}(z) \\
\frac{d A_{k}^{-}}{d z}=j \hat{\sigma} A_{k}^{-}(z)+j \kappa A_{k}^{+}(z)
\end{gathered}
$$

Where $\hat{\sigma}$ and $\kappa$ are represents the dc and ac coupling wave coefficients, which are defined as $[1,2,19,22]$

$$
\hat{\sigma}=\delta+\sigma-\frac{1}{2} \frac{d \phi}{d z}
$$

$$
\kappa=\kappa^{*}=\frac{\pi}{\lambda} \nu \bar{\delta} n_{e f f}
$$

where $\bar{\delta} n_{\text {eff }}$ is represents the dc change in the effective fiber-core refractive index, $v$ is the fringe visibility and $\varphi(z)$ represents the fiber gratings chirp. In Equation 6, the detuning $\delta$ and the $\sigma$ factors are defined as $[1,2$, $19,22]$

$$
\delta=\beta-\frac{\pi}{\Lambda}=2 \pi n\left(\frac{1}{\lambda}-\frac{1}{\lambda_{B}}\right)
$$

$\sigma=\frac{2 \pi}{\lambda} \bar{\delta} n_{e f f}$

By solving equations (4) and (5) using the transfermatrix method, the reflectivity of a uniform FBG model shown in Fig. 1 is given by $[1,2,19,22]$

$$
r=\frac{\sinh ^{2}\left(\sqrt{\kappa^{2}-\hat{\sigma} L_{g}}\right)}{\cosh ^{2}\left(\sqrt{\kappa^{2}-\hat{\sigma} L_{g}}\right)-\frac{\hat{\sigma}^{2}}{\kappa^{2}}}
$$




\section{SENSING MEChANISMS}

The main elements that influence and control the FBG properties are the $L_{g}$, the $\Lambda$ and the $n_{\text {eff. }}$. In contrast, the basic parameters of the FBG sensor are temperature, strain, and pressure, respectively. Therefore, the FBG sensor mechanism is based on observing the shift in the $\lambda_{B}$ with changing in any of these parameters $[1,2]$.

\section{A. First mechanism: By Changing the Temperature}

When a change in the temperature $\Delta T$ is happened, a shift in Bragg wavelength $\Delta \lambda_{B}$ will be occur and can describe by $[1,2]$

$$
\Delta \lambda_{B}=\lambda_{B}(\alpha+\xi) \Delta T
$$

Where,

$$
\begin{gathered}
\alpha=\frac{1}{\Lambda}\left(\frac{\partial \Lambda}{\partial T}\right) \\
\xi=\frac{1}{n_{\text {eff }}}\left(\frac{\partial n_{e f f}}{\partial T}\right)
\end{gathered}
$$

\section{B. Second mechanism: By changing the Strain}

By changing the applied longitudinal strain $\Delta_{\varepsilon z}$, the Bragg wavelength will be shifting $\Delta \lambda_{B}$ by $[1,2]$

$$
\Delta \lambda_{B}=\lambda_{B}\left(1-P_{e}\right) \varepsilon_{z}
$$

In Eq. 14, $P_{e}$ is the effective strain-optic constant which is given by $[1,2]$

$$
P_{e}=\frac{n^{2}{ }_{e f f}}{2}\left[P_{12}-v\left(P_{11}+P_{12}\right)\right]
$$

In Eq. $15, P_{11}, P_{12}$ and $v$ are the fiber optic strain components and the Poisson's ratio, respectively [1, 2].

\section{Third mechanism: By changing the Pressure}

The shift in the $\lambda_{\mathrm{B}}$ due to the change in the applied pressure can be defined by $[1,2]$

$$
\Delta \lambda_{B}=\lambda_{B}\left[-\frac{(1-2 v)}{E}+\frac{n^{2} \text { eff }}{2 E}(1-2 v)\left(2 P_{12}+P_{11}\right)\right] \Delta P
$$

In Eq. 16, $E$ is represents the FBG Young's modulus.

\section{RESULTS and DISCUSSION}

Figure 2 show the effect of grating length $\left(L_{g}\right)$ on the FBG reflectivity characteristics at constant temperature (i.e. at room temperature [1]). As shown, when $L_{g}=1$ $\mathrm{mm}$, the FWHM is about $1.6 \mathrm{~nm}$. Then it has reduces to $1.52 \mathrm{~nm}$ by increasing the $L_{g}$ value to the $2 \mathrm{~mm}$. Further increasing in the $L_{g}$ value to the $3 \mathrm{~mm}$ leads to reduce the FWHM again to the $1.4 \mathrm{~nm}$. More reduction has been happened by increasing the $L_{g}$ value to the $5 \mathrm{~mm}$, where the FWHM has reduced to the $1.28 \mathrm{~nm}$. However, this behavior of decreasing the FWHM with the increasing of the $L_{g}$ value does not continue, where for $L_{g} \geqq 7$ the is no significant effect on the FWHM as shown in Fig. 3 and is maintained approximately at 1.0 $\mathrm{nm}$ for more increasing in the $L_{g}$ value until $10 \mathrm{~mm}$. This is because the FBG reflectivity was increased to reach an approximately $85 \%$ of its maximum value with an increase in $\mathrm{Lg}$ value from 1 to 7 , as is evident in Fig. 2.
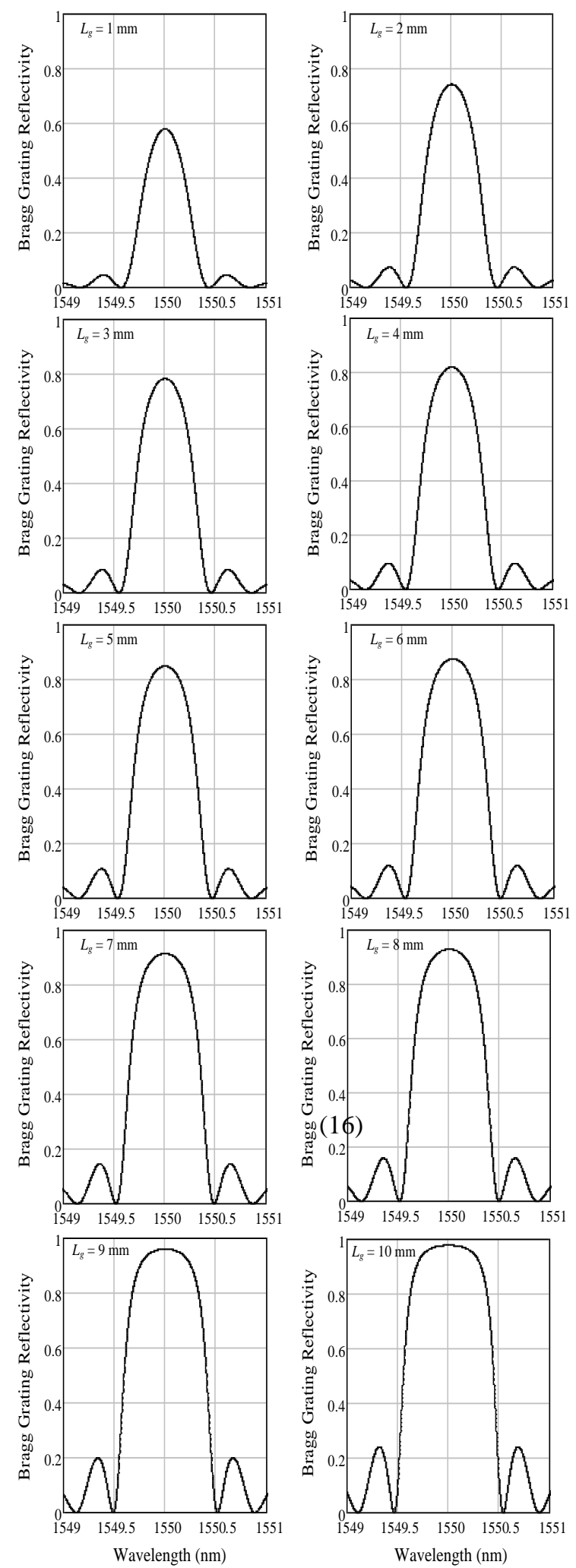

Fig. 2: Effect of grating length $\left(L_{g}\right)$ on FWHM characteristics of FBG sensor 


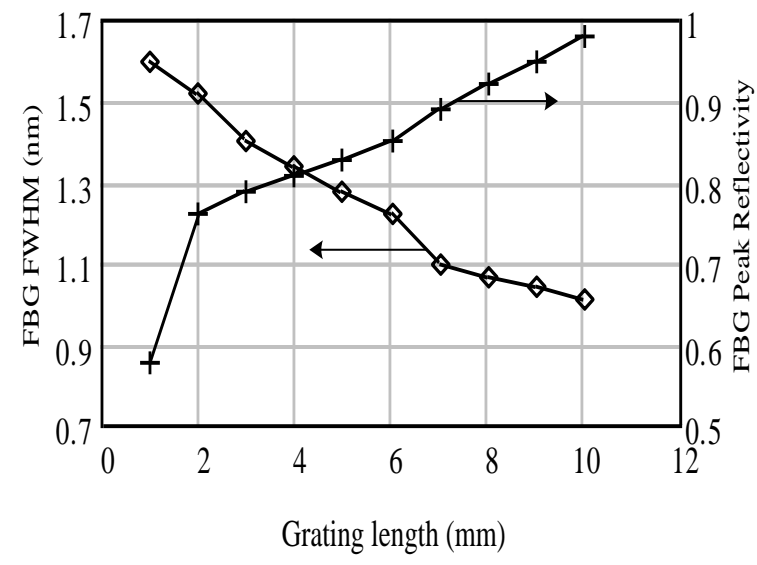

Fig. 3: Effect of grating length $\left(L_{g}\right)$ on both the FBG FWHM and the peak reflectivity

Figure 4 show the effect of temperature $(T)$ variation on the FBG FWHM characteristics for $L_{g}=10 \mathrm{~mm}$. In this analysis, $T$ has varied from $10{ }^{\circ} \mathrm{C}$ to $T_{o}+10{ }^{\circ} \mathrm{C}$ (i.e. $T_{o}$ is assumed as a reference temperature (i.e. $T_{o}=25^{\circ} \mathrm{C}$ ) $[1$, $6,7,8]$ ). As shown, the changing in temperature value leads to a shift in the Bragg wavelength by a rate of a $0.12 \mathrm{~nm} /{ }^{\circ} C$. This shifting is due the strong dependence of the reflected wavelength on the grating fiber refractive index; and the latter is a temperature depended (TD) parameter $[1,2,6,7,8]$. This shifting whether from the left-or-from the right of the center reflected wavelength (i.e. $\lambda_{\mathrm{g}}$ ) affect significantly on the peak reflected value. On the other hand, there is no significant or clear effect on the FBG FWHM characteristics as shown in Fig. 5 and in Fig. 6. The reason for this behavior is due to the fact that the change in the temperature resulting in a total shift in the reflectivity spectrum, thereby; the distance between the first two zeros in the spectrum do not change. On the other hand, these results (i.e. in Fig. 5) are totally consistent with the results obtained in the previous figure about the effect of grating length, where results showed that for $L_{g} \geq 7 \mathrm{~mm}$, the FBG FWHM approximately $1 \mathrm{~nm}$.

Finally, Fig. 7 shows the effect of the amplitude of the refractive index change $(\Delta n)$ on the FBG FWHM characteristics for $L_{g}=10 \mathrm{~mm}$. As shown, the FWHM value is increases approximately linearly with the increase of the $\Delta \mathrm{n}$ value especially for $\Delta n \geq 0.4 \times 10$ 4.This behavior is used for calculating the external perturbations like temperature, strain, pressure etc.

\section{CONCLUSIONS}

The effects of the grating length $\left(L_{g}\right)$, the amplitude of the refractive index change $(\Delta n)$ and the temperature ( $T$ ) variation on the full width half maximum (FWHM) response of a uniform fiber Bragg grating (FBG) as a sensor device for petroleum applications are investigated numerically using MATHCAD software successfully. Results show that the FWHM value effected significantly by the $L_{g}$ and the $\Delta n$ values. Thus, for a maximum FBG reflectivity and large FWHM value, the $L_{g}$ must be optimized and the $\Delta n$ must be as large as possible. In contrast, there is no significant effect on temperature variation on the FWHM value.
These results are providing a clear understanding for the FBG behavior as an important sensor device.
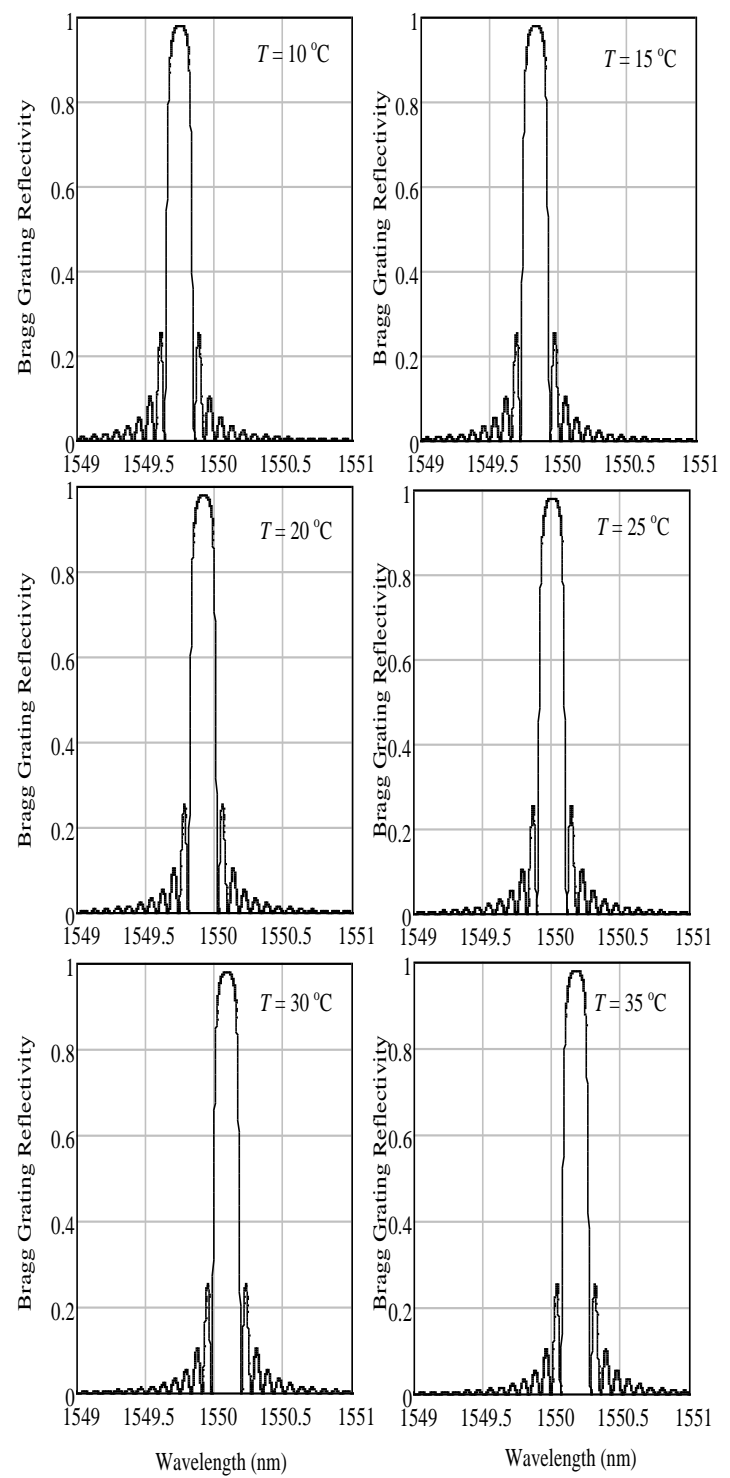

Fig. 4: Effect of temperature variation on the FBG reflectivity characteristics

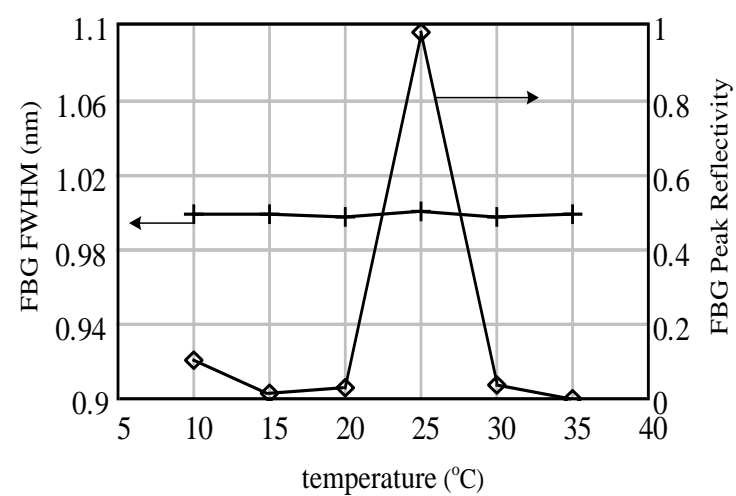

Fig. 5: Effect of temperature variation on both the FBG FWHM and the peak reflectivity 


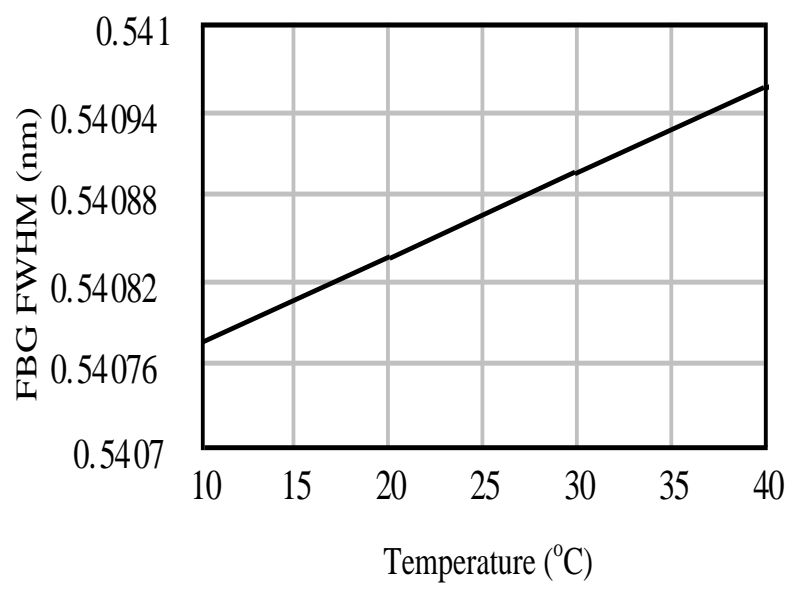

Fig. 6: Effect of temperature variation on the FBG FWHM characteristics

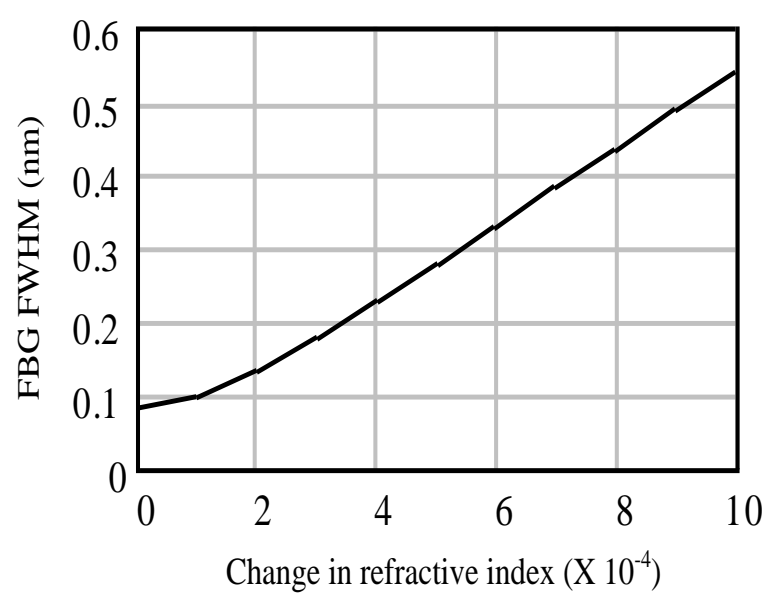

Fig. 7: Effect of refractive index change $(\Delta n)$ on the FBG FWHM characteristics.

\section{CONFLICT OF INTEREST}

The authors have no conflict of relevant interest to this article.

\section{REFERENCES}

[1] Hisham K. Hisham, Fiber bragg grating sensors: development and applications, CRC Taylor \& Frances, 2019, $1^{\text {st }}$ edition.

[2] A. Othonos, and K. Kalli, Fiber bragg gratings: fundamentals and applications in telecommunications and sensing, 1999, Artech House, Norwood.

[3] P. G. E. Lumens, "Fibre-optic sensing for application in oil and gas wells", P.hD thesis, Eindhoven University of Technology, 2014.

[4] A. C. Gringarten, et al, "Use of Downhole Permanent Pressure Gauge Data to Diagnose Production Problems in a North Sea Horizontal Well," SPE Conference, 2003.

[5] T. Unneland ,'Permanent Downhole Gauges Used in Reservoir Management of Complex North Sea Oil Fields," SPE, 1994.

[6] Hisham K. Hisham, "Numerical Analysis of Thermal Dependence of the Spectral Response of
Polymer Optical Fiber Bragg Gratings", Iraq J. Electrical and Electronic Engineering, vol.12, pp.85-95, 2016.

[7] Hisham K. Hisham, "Effect of Temperature Variations on Strain Response of Polymer Bragg Grating Optical Fibers", Iraq J. Electrical and Electronic Engineering, vol. 1, pp.53-58, 2017.

[8] H. K. Hisham," Theory of Dispersion Reduction in Plastic Optical Gratings Fiber", J. Opt. Commun., V. 9, pp.2-4, 2018.

[9] A. M. B. Braga, "Optical Fiber Sensors for the Oil and Gas Industry," in Advanced Photonics, Optical Society of America, 2014.

[10] C. E. Campanella, at al, "Fibre Bragg Grating Based Strain Sensors: Review of Technology and Applications", Sensors, vol.. 18, pp. 31-51, 2018.

[10] X. Qiao, Z. et al, "Fiber Bragg Grating Sensors for the Oil Industry”, Sensors, vol.. 17, pp. 33-49, 2017.

[11] G. Allwood, et al, "Fiber Bragg Grating Sensors for Mainstream Industrial Processes", Electronics, vol. 6, pp. 83-92, 2017.

[12] S. R. K. Morikawa, et al, "Monitoring of flexible oil lines using FBG sensors", Proceedings of SPIE The International Society for Optical Engineering, 2007.

[13] Z. Sun, et al, "A High Sensitivity Fiber Bragg Grating Seismic Sensor System for Intrusion Detection", International Symposium on Photonics and Education in Measurement Science, 2019, Jena, Germany.

[14] J. Dorleus, et al, “A Fiber Optic Seismic Sensor for Unattended Ground Sensing Applications", ITEA Journal, vol.. 30, pp. 455-460, 2009.

[15] L. F. Mennella, et al, "A Fiber Optic Bragg Grating Seismic Sensor", IEEE Photonics Technology Letters, vol. 19, pp. 1991 - 1993, 2008.

[16] J. Wang, et al, "Fiber Bragg Grating (FBG) Sensors Used in Coal Mines", PHOTONIC SENSORS, vol.. 4, pp. 120-124, 2014.

[17] Y. Zhang, et al, "Seismic wave detection system based on fiber optic sensor", Proc. SPIE. Earth Observing Systems, September 2006.

[18] Y. R. Garc, et al, "Vibration Detection Using Optical Fiber Sensors”, Journal of Sensors, vol. 12, pp. 11-55, 2010.

[19] Hisham K. Hisham, "Bandwidth Characteristics of FBG Sensors for Oil and Gas Applications", American Journal of Sensor Technol., vol. 4, pp. 3034, 2017.

[20] D. J. M Snelders and A. Boersma, "Development of thermo-stable FBG optical sensor for oil and gas applications", Proc. of the 8th Inter. Conf. on Sensing Technolo., pp. 278-281, 2014, UK.

[21] K. T. V. Grattan and T. Sun, "Fiber optic sensor technology: an overview," Sensors and Actuators A: Physical, vol. 82, pp. 40-61, 2000.

[22] G. P. Agrawal and N. K. Dutta, Semiconductor Lasers, $2^{\text {nd }}$ ed. (New York: van Nostrand Reinhold, 1993). 